\title{
Left Main Coronary Artery Dissection during Breastfeeding Treated with CABG Complicated by Severe Ventricular Disfunction and Left Ventricular Thrombosis
}

\author{
Nicolò Sisti, MD*, Giulia Elena Mandoli, MD, Antonio Pagliaro, MD, Giuseppe \\ Guerrieri, MD, Carlotta Sciaccaluga, MD, Matteo Cameli, MD, PhD, Sergio \\ Mondillo, MD and Serafina Valente, MD
}

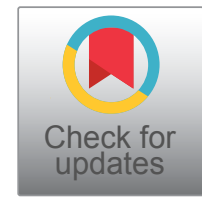

Department of Cardiovascular Diseases, University of Siena, Italy

*Corresponding author: Nicolò Sisti, MD, Department of Cardiovascular Diseases, University of Siena, Siena, Italy

\begin{abstract}
Spontaneous coronary artery dissection (SCAD) should always be suspected in presence of chest pain during post-partum, since it is one of the possible causes. Strategies for the management of such disease and its complication are not still clearly encoded. A 40-years-old woman was referred with chest pain, started while breastfeeding, associated with an EKG pattern suggesting ischemia. After hospital admission, coronary angiography was performed showing a Type 1 left main coronary dissection. Given persistent symptoms, hemodynamic instability and persistent EKG alterations the patient underwent urgent coronary artery bypass grafting (CABG). Post-surgical period was complicated by severely reduced left ventricular systolic function with secondary pulmonary hypertension, requiring inotropic support and inhaled nitric oxide, respectively. Moreover, apical thrombosis was detected which promoted the adoption of an anticoagulation strategy. Management of lactation was also necessary. Left ventricular dysfunction persisted at discharge and despite prognostic data were in favour of optimal functional recover, the patient was scheduled for a cardiac magnetic resonance for further evaluation and therapeutic assessment.
\end{abstract}

\section{Introduction}

Spontaneous coronary artery dissection (SCAD) predominantly affects the female population, involving women in more than $90 \%$ of cases and being the cause of acute myocardial infarction (AMI) in $24 \%$ of women with AMI under the age of 50 [1]. In pregnancy subgroups, SCAD is responsible of acute myocardial infarction in $43 \%$ of cases among women with an average age of 34, during third trimester or in the immediate postpartum. According to the original definition, it is not induced by trauma or atherosclerosis [2,3]. As described in our case, the clinical onset is typically chest pain, and the impact on left ventricular function might be variable, showing an overall better prognosis if compared to the other causes of AMI [4]. The optimal treatment of this condition is still not clearly defined, requiring a balanced assessment between the probability of spontaneous healing and the existing complications.

\section{Case Presentation}

A 40-year-old woman, in her $4^{\text {th }}$ month of postpartum, second pregnancy, presented to a peripherical medical aid complaining of stabbing epigastric pain radiated to the interscapular region, started about 1 hour earlier while breastfeeding. She referred having similar symptoms 20 days before, similarly induced by breastfeeding, solving spontaneously in about $15 \mathrm{~min}$ utes. Cardiovascular risk factors were absent, and her family history regarding heart diseases was silent. The previous pregnancy was described free of complications during both pre-partum and post-partum.

Physical examination did not add pathological details. Respiratory rate was $16 / \mathrm{min}$. Normal S1 and S2 heart sounds were present, no murmurs or gallops were detected. There were no signs of heart failure visible. The pain was constant, independent from both decubitus and respiratory movements. Measured arterial 


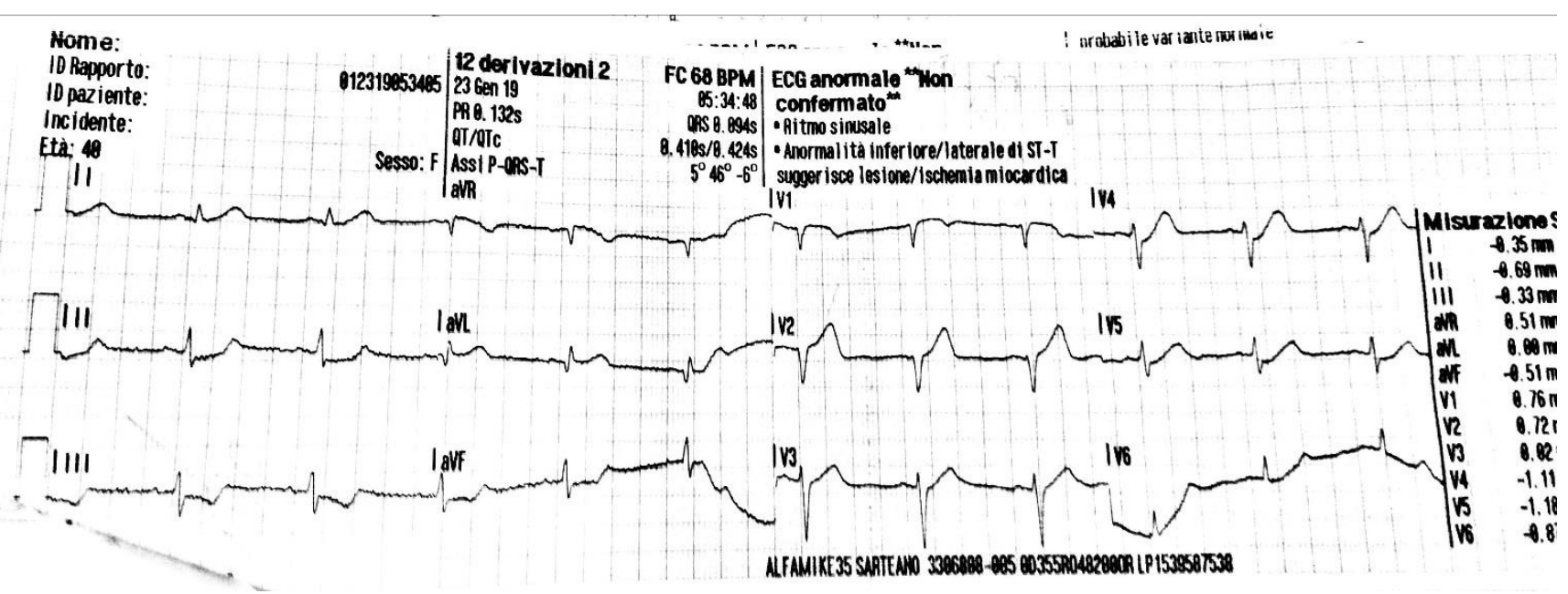

Figure 1: EKG presentation.

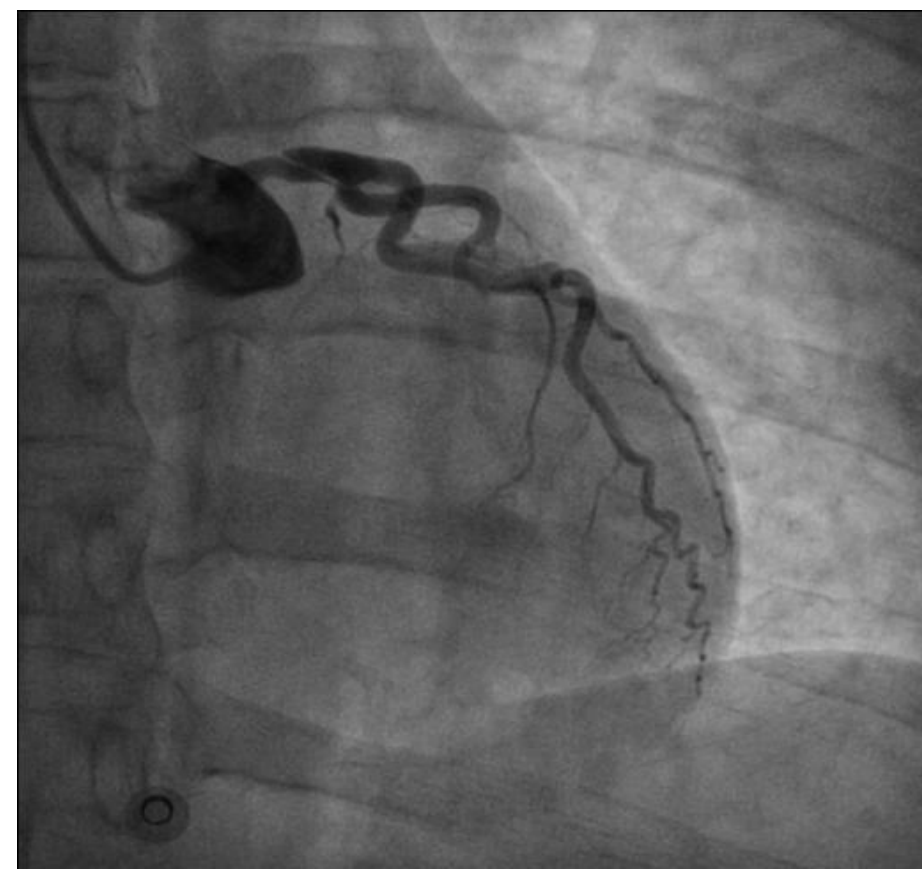

Figure 2: Coronary angiography showing spontaneous left main coronary artery dissection.

pressure was $95 / 70 \mathrm{mmHg}$, while peripheral oxygen saturation was $95 \%$. EKG revealed sinus rhythm at 75 bpm, normal AV conduction, $1 \mathrm{~mm}$ ST downslope in D2D3-aVF and V4V5-V6, $1 \mathrm{~mm}$ ST elevation in aVR and 1.5 $\mathrm{mm}$ in V1-V2, delayed R-wave progression from V1 to V4 (Figure 1). Suspecting an acute coronary syndrome (ACS) or an acute aortic disease, our Cath Lab (about one hour's drive distant) was contacted and the transfer was activated. General treatment measures were initiated: Lysine Acetylsalicylate $500 \mathrm{mg}$ e.v., intravenous infusion of sodium chloride solution and proton pump inhibitor e.v. During the transfer to our hospital, arterial pressure persisted on low thresholds, while chest pain maintained the same characteristics with lower intensity. Emergent coronary angiography through radial approach was performed at the arrival in our hospital, and it showed TYPE 1 left main coronary dissection with TIMI 2 flow in left anterior descending coronary artery and left circumflex artery, while no angiographically significant stenosis were detected. Right coronary artery showed good canalization (Figure 2). EKG pattern presented a partial improvement at the end of the procedure. In light of the angiographical result and the hemodynamical instability, considering that arterial pressure gradually decreased reaching values below $85 / 60 \mathrm{mmHg}$, we opted for positioning an intra-aortic balloon pump (IABP) 1:1 through right femoral access, sending then the patient to surgical revascularization. Pre-operative blood gas analysis showed $\mathrm{pH} 7.39, \mathrm{pO}_{2}$ $177.5 \mathrm{mmHg}$ with $\mathrm{FiO}_{2} 50 \%, \mathrm{pCO}_{2} 32.6 \mathrm{mmHg}, \mathrm{HCO}_{3}-20$ $\mathrm{mmol} / \mathrm{L}$, Lactates $0.59 \mathrm{mmol} / \mathrm{L}, \mathrm{K}^{+} 3.3 \mathrm{mmol} / \mathrm{L}$. In the operating room, a pre-surgical trans-oesophageal echocardiography was performed and it revealed a global left ventricle hypokinesis with marked hypo-akinesis of interventricular septum. The patient underwent onpump double coronary artery bypass grafting (CABG) 


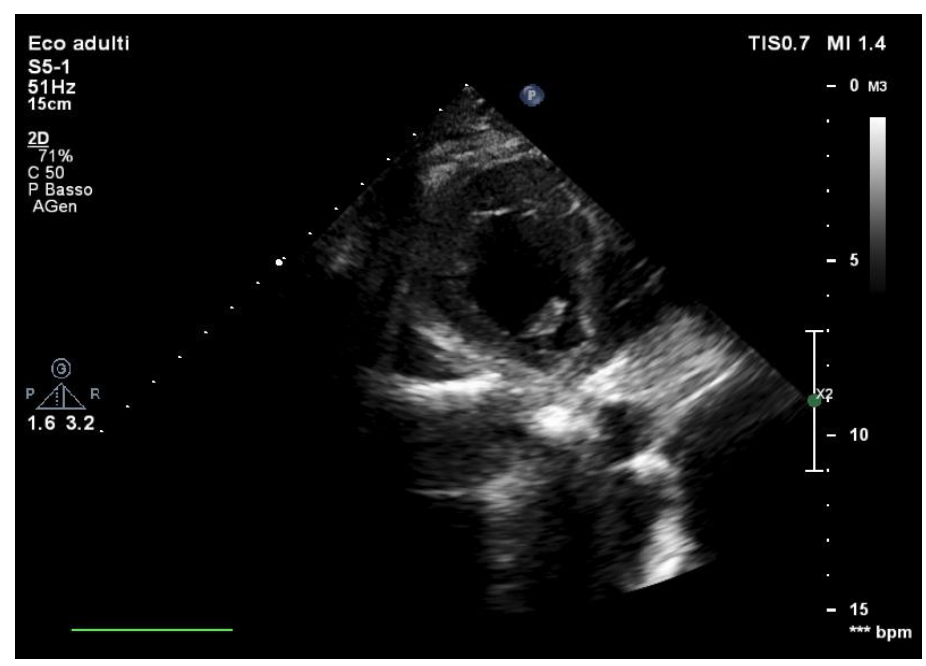

Figure 3: Transthoracic PSAX view of thrombotic stratification in left ventricle.

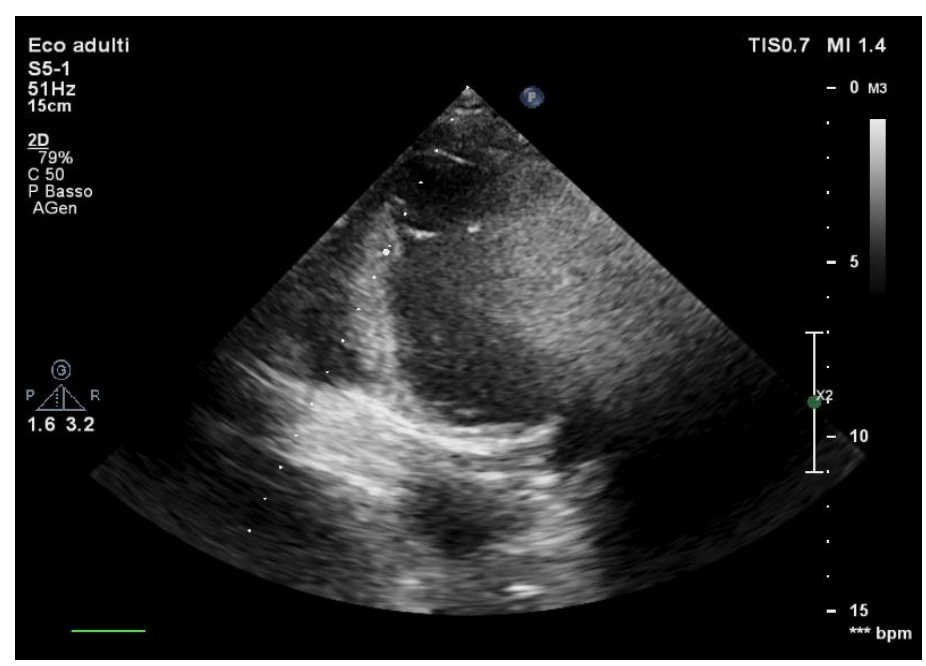

Figure 4: Transthoracic apical 2 chamber view of the aberrant chord.

with anastomosis between internal mammary artery and left anterior descending coronary and between saphenous graft and obtuse marginal branch. After the surgery hemodynamics was supported with IABP 1:1, Adrenaline 0.2 micrograms $/ \mathrm{kg} / \mathrm{min}$ and Noradrenaline $0.1 \mathrm{micrograms} / \mathrm{kg} / \mathrm{min}$ infusion, Levosimendan was also administered in a $24 \mathrm{~h}$ infusion at 0.05 micrograms/ $\mathrm{kg} / \mathrm{min}$. Troponin levels measured 8 hours after the procedure were $7027 \mathrm{pg} / \mathrm{ml}$. In addition, Cabergoline 0.25 mg every 12 hours for 48 hours was considered, with the aim to decrease lactation.

Post-procedural transthoracic echocardiography (TTE) showed a left ventricular ejection fraction (LVEF) of $20 \%$, akinesia of interventricular septum, apical segments and left ventricular lateral wall and marked hypokinesis of the mid anterior wall. Right ventricular chamber resulted to be slightly dilated and pulmonary artery systolic pressure (PAPs) was roughly $50 \mathrm{mmHg}$. Inferior vena cava appeared dilated without respiratory collapse. Valvular apparatus presented no defects and pericardial effusions was absent. A chest $X$ Ray did not detect any abnormalities. In consideration of signs of pulmonary hypertension, also associated with elevated transaminases levels (GPT $73 \mathrm{UI} / \mathrm{I}$, GOT $213 \mathrm{UI} /$ ), inhaled nitric oxide 20 ppm was added to the therapeutic approach. An episode of asymptomatic atrial fibrillation with elevated ventricular response was interrupted through a intravenous bolus of Amiodarone.

During the third day after the event, the patient was weaned from mechanical ventilation and a TTE confirmed an improvement of systolic function with LVEF $30 \%$, however no variations of kinesis abnormalities were found. Persistent good values of arterial blood pressure allowed a gradual decalage and suspension of intravenous inotropes as well as IABP removal. In the fifth day, a routine TTE revealed a thrombotic stratification in apical region and inferior apical wall of approximately $1.5 \mathrm{~cm} \times 1.5 \mathrm{~cm}$ (Figure 3). At this point, it was necessary to exclude another possible diagnosis, such as the implant of an aberrant tendinous chord (Figure 4) linking the anterior and posterior apical segments, then, once the diagnosis of intra-cardiac thrombus was confirmed, unfractionated heparin infusion was started maintaining a range of aPTT between $50-70$ s. In the 
following two days echocardiographic follow up ascertained a significant reduction of the thrombosis reaching a complete resolution, then bridging with warfarin was initiated until INR $>2$.

The patient was discharged 15 days after the event. At that time, TTE documented LVEF 35\%, akinesis of interventricular septum and apical segments, hypokinesis of mid anterior wall. Right ventricular dimension and pulmonary pressure were normal. Therapy at discharge was based on loop diuretic, betablocker, angiotensin receptor blockers, potassium sparing diuretic, ivabradine and warfarin.

A cardiac magnetic resonance (CMR) was scheduled 40-days after discharge to assess in detail residual ventricular function and potential scar. Normal ventricular volumes were confirmed, LVEF was $41 \%$ with hypokinesis of mid-apical anterior wall and mid-apical interventricular septum. Myocardial edema persisted in anterior wall and anterior septum. A light subendocardial delayed gadolinium enhancement was visible in anterior wall, mid anteroseptum, mid-apical septum.

\section{Discussion}

Pregnancy related SCAD represents less than $5 \%$ of all the documented events in contemporary series and left main variants as occurred in our patient represent $36 \%$ of cases $[1,2,5]$. According to Heefner hypothesis [6], the dissection results from a "double hit" phenomenon: the first one given by hemodynamic stress during pregnancy, increased cardiac output and blood volume responsible of intimal weakening of vessels, then the second one represented by an altered hemostasis during postpartum which could pave the way to media infiltration. Also it has to be considered that according to some hypothesis, estrogen receptors expressed in coronaries wall could enhance modifications during pregnancy, like elastic fibers fragmentation, cystic necrosis of media and loss of vasa vasorum [7,8]. These changes, parallel to those already described, could trigger the dissection. Age over 30 years, hypertension, diabetes, smoking, preeclampsia, eclampsia, infections and multiparity have already been ascertained as independent risk factors $[2,9,10]$. A tight link with breastfeeding has also been proved with a direct correlation with its duration $[11,12]$. Medical therapy is targeted to stable patients with single vessel involvement and observational studies have already proved that from $73 \%$ up to $97 \%$ of SCAD are spontaneously healed beyond one month [13-15]. Revascularization must be considered according to clinical presentation and symptoms persistency, ventricular arrythmia, hemodynamic instability, site of dissection and number of involved vessels. CABG remains the indicated choice in case of multivessel dissections or left main coronary involvement, unsuitable anatomy for $\mathrm{PCl}$ or presence of signs of ischemia associated to hemodynamic instability. In more than $40 \%$ of pregnant SCAD patients, dissections could be demonstrated in more than one vessel [16]. A recent analysis based on 120 case reports of SCAD during pregnancy from 2000 to 2015 stated that CABG in that study population represented the choice of revascularization with over 20 fold increased rate when compared with nonpregnant women with $\mathrm{MI}$, and more than 10 fold when compared with nonpregnant women with SCAD. Choice of CABG in this study was guided by complex coronary anatomy (left main and multivessel involvement), failure of $\mathrm{PCl}$ and hemodynamic instability [17]. A noteworthy aspect is that long term results of CABG in SCAD are less optimal than other conditions because of a recover of the flow in the dissected artery which produce a competitive flow with the graft leading to thrombosis [18]. In our case, persistent angina since first medical contact, left main involvement and hemodynamic instability allowed us to categorize the patient as "high risk". In particular, the presence of low blood pressure was also a key point to start inotropic therapy during post-surgery and to introduce IABP as advanced support. As outlined by the expert consensus of American Heart Association [19], when indication to urgent $\mathrm{PCl}$ or CABG is confirmed, the final decision should be individualized and contemplated in the context of the coronary anatomy and the expertise of the operators or centers, which explains the need of a complete Heart Team to define the strategy. The complications that our patient faced such as pulmonary hypertension and ventricular thrombosis are as common as in a typical post-extended IMA period, moreover, as showed by Ito, et al. in a sample of 23 SCAD, postpartum patients tend to have larger infarcts and lower mean LVEF (34\% vs. 49\%; $\mathrm{P}<0.01$ ). Postpartum women also tend to have proximal artery dissections ( $86 \%$ vs. $19 \%$; $P<0.004$ ) [20].

Anticoagulant therapy, even if necessary for thrombus resolution, increases the probability for the intramural hematoma to extend and should be pursued for the shortest period as possible, as secondary prevention. Even if recent studies have successfully compared the use of direct oral anticoagulants (DOAC) and warfarin in the treatment of left ventricular thrombosis $[21,22]$ their use in this setting is still off-label and, additionally, DOAC SPCs recommend against their use in pregnancy and during breastfeeding.

\section{Conflicts of Interests}

The author declares that there is no conflict of interest regarding the publication of this paper.

\section{References}

1. J Saw, E Aymong, GBJ Mancini, Sedlak T, Starovoytov A, et al. (2014) Non-atherosclerotic coronary artery disease in young women. Can J Cardiol 30: 814-819.

2. F Alfonso, M Paulo, $V$ Lennie, Dutary J, Bernardo E, et al. (2012) Spontaneous coronary artery dissection: longterm follow-up of a large series of patients prospectively managed with a "conservative" therapeutic strategy JACC Cardiovasc Interv 5: 1062-1070. 
3. J Saw, E Aymong, T Sedlak, Buller CE, Starovoytov A, et al (2014) Spontaneous coronary artery dissection: Association with predisposing arteriopathies and precipitating stressors and cardiovascular outcomes Circ Cardiovasc Interv 7: 645-655.

4. Franco C, Starovoytov A, Mancini GB (2015) Spontaneous resolution of LV dysfunction post SCAD: A case series. SCAI 6-9.

5. S Rogowski, MT Maeder, D Weilenmann, Haager PK, Ammann P, et al. (2015) Spontaneous coronary artery dissection: Angiographic follow-up and long-term clinical outcome in a predominantly medically treated population. Catheter Cardiovasc Interv 89: 59-68.

6. Heefner WA (1973) Dissecting hematoma of the coronary artery: a possible complication of oral contraceptive therapy. JAMA 223: 550-551.

7. Elkayam U, Jalnapurkar S, Barakkat MN, Khatri N, Kealey AJ, et al. (2014) Pregnancy-associated acute myocardial infarction: A review of contemporary experience in 150 cases between 2006 and 2011. Circulation 129: 1695-1702.

8. Briguori C, Bellevicine C, Visconti G, Focaccio A, Aprile V, et al. (2010) In vivo histological assessment of a spontaneous coronary artery dissection. Circulation 122: 1044-1046.

9. R Vijayaraghavan, S Verma, N Gupta, Saw J (2014) Pregnancy-related spontaneous coronary artery dissection Circulation 130: 1915-1920.

10. PT Koller, CM Cliffe, DJ Ridley (1998) Immunosuppressive therapy for peripartum-type spontaneous coronary artery dissection: case report and review Clin Cardiol 21: 40-46.

11. Naderi S (2017) Spontaneous Coronary Artery Dissection and Pregnancy. Curr Treat Options Cardiovasc Med 19: 69

12. MH Labbok (2001) Effects of breastfeeding on the mother Pediatr. Clin North Am 48: 143-158.
13. Phillips LM, Makaryus AN, Beldner S, Spatz A, SmithLevitin $M$, et al. (2006) Coronary artery dissection during pregnancy treated with medical therapy. Cardiol Rev 14: 155-157.

14. J Saw (2013) Spontaneous coronary artery dissection. Can J Cardiol 29: 1027-1033.

15. C Lettieri, D Zavalloni, R Rossini, Morici N, Ettori F, et al. (2015) Management and long-term prognosis of spontaneous coronary artery dissection Am J Cardiol 116: 66-73.

16. Havakuk O, Goland S, Mehra A, Elkayam U (2017) Pregnancy and the risk of spontaneous coronary artery dissection: An Analysis of 120 Contemporary Cases. Circ Cardiovasc Interv 10: e004941.

17. MS Tweet, SN Hayes, SR Pitta, Simari RD, Lerman A, et al. (2012) Clinical features, management and prognosis of spontaneous coronary artery dissection. Circulation 126: 579-588.

18. Yip A, Saw J (2015) Spontaneous coronary artery dissection-A review. Cardiovasc Diagn Ther 5: 37-48.

19. Hayes SN, Kim ESH, Saw J, Adlam D, Arslanian-Engoren C, et al. (2018) Spontaneous coronary artery dissection: Current state of the science: A Scientific statement from the american heart association. Circulation 137: e523-e557.

20. Tanis W, Stella PR, Kirkels JH, Pijlman AH, Peters RH, et al. (2008) Spontaneous coronary artery dissection: Current insights and therapy. Neth Heart J 16: 344-349.

21. Ito Hiroki, Taylor L, Bowman M, Fry ET, Hermiller JB, et al. (2011) Presentation and therapy of spontaneous coronary artery dissection and comparisons of postpartum versus nonpostpartum cases. Am J Cardiol 107: 1590-1596.

22. Austin Robinson, Benjamin Ruth, John Dent (2018) Direct oral anticoagulant compared to warfarin for left ventricular thrombi: A single center experience. JACC 71: A981 